\title{
The power of two: lessons from a scientific partnership
}

\author{
Robert V. Farese Jr. ${ }^{1,2,3}$ and Tobias C. Walther ${ }^{1,2,3,4}$ \\ 'Department of Molecular Metabolism, Harvard T.H. Chan School of Public Health, Boston, Massachusetts, USA. ²Department of Cell Biology, Harvard Medical School, Boston, Massachusetts, USA. ${ }^{3}$ Broad \\ Institute of Harvard and MIT, Cambridge, Massachusetts, USA. ${ }^{4}$ Howard Hughes Medical Institute, Boston, Massachusetts, USA.
}

M dern science is a collaborative effort. Scientists no longer toil in solitude in the dark corner of a laboratory seeking knowledge. As collaboration increases, running a joint laboratory, as in the celebrated scientific partnerships of Carl and Gerty Cori $(1,2)$, Mike Brown and Joe Goldstein $(3,4)$, or Mike Bishop and Harold Varmus (5), has emerged as a highly successful model for discovery. For those considering this path, we reflect upon principles that we have learned, from our mentors and in the practice of our 16-year scientific partnership, including the operation of a joint laboratory over the past six years.

\section{Why form a scientific partnership?}

History is full of creative collaboration (6, 7). We can easily measure these partnerships by their end products, but it's not always clear why and how to get started down this path. Chief among the many reasons to form a partnership is to enable more exciting science. Great science begins with a hypothesis, and creative, well-informed hypotheses are more likely to result in breakthrough discoveries. Opportunities to discuss ideas and to converse and disagree with a partner within an environment of trust, with shared foundational knowledge, and without a power differential can catalyze key questions and insights. Indeed, we relish the opportunity to brainstorm ideas on a near-daily basis with someone possessing similar "institutional knowledge," which is difficult to reproduce with a trainee or colleague. We speak the same language and can more quickly get to the heart of a problem. There is also a practical advantage - in our information-overloaded world, our collaboration also helps to fill in important details; although we each forget many things, we fortunately seem to not forget the same things!
The interplay of ideas also guards against bias: most people like their own ideas better than those of others. A hazard of science is to be so convinced of one's own hypothesis that we ignore contrary evidence. As two highly aligned individuals filter ideas, emotional distance from the ideas increases, enabling faster prioritization of good ones, discarding of poor ones, and avoiding (or more quickly recognizing) unproductive excursions.

We also take great pleasure in the joy of creative collaboration, recognizing that the outcome is uniquely different from what either person might do alone. When partners have different scientific backgrounds, as in our case, the collective experiences can bring a variety of powerful approaches for solving scientific problems. Combining our expertise in different scientific disciplines, such as biochemistry, genetics, physiology, and medicine, helps us to see problems from different angles and thus can lead to more interesting questions and a diversity of routes to solutions.

Partners also facilitate the challenges of making major decisions. Two primary challenges for any scientist are deciding what to work on and whom to hire. When leading a laboratory, the answers to these questions can make or break a career. The power of combined, invested viewpoints enables a more careful examination and therefore a higher likelihood of arriving at better answers.

With respect to hiring, our different scientific backgrounds also help us to attract and recruit a diverse group of scientists. Bob studied medicine in the US, while Tobi studied biochemistry in Germany, enabling us to provide mentoring from different viewpoints. As a result, our trainees come from all over the world, with expertise ranging from physics to physician. Through our partnership, we model scientific collaboration on a daily basis, and many people we recruit are attracted to this model.

Perhaps the most important motivation for forming a partnership is the most obvious: it is simply fun to do science with a partner. Anyone can have a great day on his or her own, but the excitement of solving problems through shared debate and discovery with someone who understands the situation and appreciates the setbacks and work that went into finding a solution can bring great joy. Conversely, bad days can be eased when you can share them with your partner, who can act as an emotional buffer. Having a partner can also help to prevent pressure from being misdirected at your trainees: it is okay to tell your science partner that you are not sure a project makes sense and then change your mind the next day. Yet such a conversation could be quite unsettling to a trainee.

\section{How do you find a partner?}

There are many paths toward finding a partner, with serendipity playing a large role. In our case, we were both strongly interested in collaborative research. Bob was primarily working on lipid metabolism and physiology, wanted to perform more cell biology research, and had been exposed early on to the impressive synergy of the Brown and Goldstein partnership. Tobi was working on cell biology and membrane biochemistry, wanted to pursue more physiology, and had enriching and impactful experiences with collaborative research during his thesis work and postdoc. We met because we were both spending time, for different reasons, in the laboratory of Peter Walter at UCSF. In this creative and playful environment, we began discussing questions that we both found fascinating: How do cells store metabolic energy as oils in lipid droplets? How does the endoplasmic reticulum give rise to these organelles? Do all cells produce lipid droplets? We 
recognized this as an exciting new frontier of cell biology and embarked together on an exploratory adventure to answer these questions. Sixteen years later, we have uncovered some answers and continue to explore vast uncharted lands.

What career stage is ideal for forming a partnership? Here, we think there are no rules, only guidelines. We have seen partnerships take off between junior investigators or more senior scientists; in all cases, a shared passion for the science has been the driver. It may help to be relative contemporaries, but it is not essential.

Perhaps the most important task is finding a partner who shares your scientific values. In our case, we always agree on what we view as important questions and outstanding science. As Brown and Goldstein said, "not seeing eye to eye" can be fatal in partnerships. Equally important as scientific compatibility is emotional compatibility. Partners must have the capacity to handle challenging situations that arise in the relationship judiciously and with empathy. Finally, there is the chemistry between partners - that vital yet intangible quality of enjoying being around someone with a compatible personality. Thus, in pursuing a partnership, look for someone who fits your values, temperament, and personality.

\section{How do you manage a joint laboratory?}

How are projects managed? Should you form a single joint laboratory or two laboratories functioning in parallel? How should responsibilities and credit be distributed? There are no right answers to these questions, of course, but we provide some insights from our experiences.

We opted to completely join forces and form one laboratory with equal responsibilities and credit. Essentially, we decided to be "all in" together, feeling that other models might lead to internal strife. We believe that this approach may be best for establishing long-lasting partnerships. However, the many variables faced by scientists may lead to other optimal solutions for creative collaboration.

In our model, we make all important decisions together and actively involve our laboratory members. Examples include decisions on scientific directions, research hypotheses, data interpretation, publication and grant application content, and hiring. In most cases, our views and approaches align well; if we do not agree, we continue conversing until we find agreement. This approach also enables us to continually educate each other about different scientific areas, methodologies, and approaches. We nurture each other's growth.

As an exception to this approach, we often provide career mentoring to trainees independently. We find that one-on-one conversations with each of us are often more useful for trainees, providing them with insights from diverse viewpoints. Nonetheless, we discuss nearly everything as partners and find that we almost always agree on the salient points of such career discussions.

For minor decisions and day-to-day operations, we usually do whatever gets the job done. One advantage of a partnership is that one can distribute managerial and administrative tasks and thus have more time for creative work. For example, we each take the lead in ensuring that different tasks are accomplished. This approach works, most of the time: it is not uncommon to hear us yell from our adjoining offices, "I thought you were doing that!"

\section{What are the major challenges to a scientific partnership?}

In our experience, unlike in personal relationships, there appears to be less chance that your scientific partner will rapidly divorce you for a perceived better mate. The work involved in establishing a scientific partnership is too great for frequent or rapid changes. Yet some forces can generate pressure on a science partnership.

For example, you must learn the ins and outs of working intensively together. Although we had run highly collaborative, individual laboratories for ten years, the situation changed when we combined laboratories and began to manage them jointly. Everyone has different habits and organizational styles; thus, it may take some time to find an optimal day-to-day routine.

Issues of credit present another challenge. Most science systems value and evaluate individuals. This approach is reflected in promotions, awards, and funding, credit for publications, and being the public face of the research. This facet can be challenging to navigate. "Ego strokes" or "ego blows" are seldom perfectly balanced. At any time, one person will experience more of one or the other, which can put strain on a partnership. Indeed, the effects of these emotional aspects are often primary reasons for dissolving creative partnerships.

How can one overcome these challenges? First, institutional buy-in to the scientific partnership model is critical. Institutions should support rather than try to divide partners at crucial steps of career development. Similarly, funding agencies often prefer recognizing individuals rather than partnerships, which can cause pressure. As the number of partnerships increases, however, increasing recognition of partnerships by institutions and funders should ease these issues.

Another important tool is branding the partnership and generating a unit that is larger than either investigator. The best approach is to achieve recognition for the partnership's product. Brown and Goldstein were awarded a Nobel prize at a rather early stage of their partnership, effectively cementing them as a team.

For mere mortals like us, we feel we must go the extra mile in our interface with the academic world. We take every opportunity to emphasize our shared laboratory and resist any temptation to assign individual credit. Our publications come from the "Farese and Walther laboratory," and we stress "Bob and Tobi" over either partner. Simultaneously, we keep in mind that a thriving partnership must value independent autonomy as well as the rewards of collaboration.

Another principle is to cheer on your partner. What goes around comes around. Sometimes, one must sacrifice a bit of one's ego to achieve greater success in the partnership and, most importantly, to make scientific progress. Each partner must be sensitive to the other's needs and provide support during challenging moments. We find that honest conversations regarding the challenges and frequent reminders of why we are in this together serve to strengthen our partnership. Over time, such habits generate trust and, hopefully, more scientific success. 


\section{Any take homes?}

We have enjoyed our scientific journey together immensely and can barely remember what science was like in the years before. Without a doubt, we have each become better scientists because of our partnership. Is the partnership model for everyone? Likely not. However, we believe that partnerships spotlight a scientific culture that values insights gained from collective inquiry rather than the culture of individuals winning or losing. Partnerships thrive on the values of respect, trust, open communication, and shared credit. "It's amazing how much can be accomplished if no one cares who gets the credit" (Harry S Truman). Maybe a bit more of that approach in science could be beneficial.

\section{Acknowledgments}

The opinions expressed here are our own, but we are grateful for advice from other scientific partnerships, including Brown and Goldstein, Hobbs and Cohen, and Mangelsdorf and Kliewer, all at UT Southwestern. We thank G. Howard and J. Gosse for editorial assistance and T. Rapoport, $\mathrm{U}$. Gritzan, and B. Schulman for comments on the article.

Address correspondence to: Robert V. Farese Jr. or Tobias C. Walther, Department of Molecular Metabolism, Harvard T.H. Chan School of Public Health, 665 Huntington Avenue, Building 1, Room 207A, Boston, Massachusetts 02115, USA. Phone: 617.432.3128; Email: robert@hsph.harvard.edu (RVF); twalther@ hsph.harvard.edu (TCW).
1. Cori CF, Cori GT. Carbohydrate metabolism. Annu Rev Biochem. 1946;15:193-218.

2. NobelPrize.org. The Nobel Prize in Physiology or Medicine 1947. https://www.nobelprize.org/ prizes/medicine/1947/summary/. Accessed December 1, 2020.

3. Anderson RG. Joe Goldstein and Mike Brown: from cholesterol homeostasis to new paradigms in membrane biology. Trends Cell Biol. 2003;13(10):534-539.

4. NobelPrize.org. The Nobel Prize in Physiology or Medicine 1985. https://www.nobelprize.org/ prizes/medicine/1985/summary/. Accessed December 1, 2020.

5. NobelPrize.org. The Nobel Prize in Physiology or Medicine 1989. https://www.nobelprize.org/ prizes/medicine/1989/summary/. Accessed December 1, 2020.

6. Shenk JW. Powers of Two: Finding the Essence of Innovation in Creative Pairs. Eamon Dolan/ Houghton Mifflin Harcourt; 2014.

7. John-Steiner V. Creative Collaboration. Oxford University Press; 2000. 